\title{
An Epidemic Spreading Model on the Adaptive Dual networks
}

\author{
Changlun Zhang ${ }^{1}$, Nan Ning ${ }^{1}$ Zhanyong Jin $^{2}$, Jian Zhang ${ }^{1}$ \\ ${ }^{1}$ (The school of science, Beijing University of Civil Engineering and Architecture, China) \\ ${ }_{2}^{2}$ (The school of economics and management engineering, Beijing University of Civil Engineering and \\ Architecture, China)
}

\begin{abstract}
Information diffusion and disease spreading in awareness-contact layered network are typically asymmetrically coupled with each other, in which how an individual being aware of disease responds to the disease can significantly affect the epidemic spreading. During the two processes above, there will be an adaptive evolution machine in which the diffusion of the disease or the information will lead individual to break or generate the link to avoid infecting the disease or to get more information, and then the link changes will react to the diffusion processes. In this paper, we carry out an epidemic spreading model on the adaptive dual network based on the machine to study the interplay between the two processes. By constructing two adaptive networks, we consider a new link breaking phenomenon on the epidemic spreading process and the heterogeneity of the link breaking probability. The simulation results show that our model will slow down the disease spreading and reduce the infected size. In addition, the homogeneous of the link breaking probability will increase the epidemic threshold.
\end{abstract}

Keywords: epidemic spreading, community structure, dual network, information communication

\section{INTRODUCTION}

When a disease suddenly emerges, there is an asymmetric coupling process between the disease awareness diffusion and the epidemic spreading [1-5]. In particular, the spread of diseases results in elevated crisis awareness and thus facilitates the diffusion of the awareness about the disease [6], but the diffusion of the awareness promotes more people to take preventive measures and consequently suppresses the epidemic spreading [4]. To understanding how awareness diffusion can mitigate epidemic outbreaks, and more broadly, the asymmetric interacting spreading dynamics led to a new direction of research in complex network science [7-14]. A pioneering step in this direction was taken by Clara [7] who studied the epidemic spreading in the multiplex networks by establishing two layers network, in which one represents epidemic spreading and another represents the diffusion of the information awareness. And the results showed that there is a critical point at which the disease spreading can be controlled by the individual information awareness. Usually, the attributes of the nodes and the links on the network are different. In view of this, the scholars have embodied the dual network epidemic model. Liu et al. [9] studied the effect of the community size on the two interplay dynamics, and found that the larger the community size is, the faster the epidemic spreading outbreaks. Guo et al [14] carried out a multiplex network model based on the activity-driven pattern by considering the social network is a time-varying network, and the results show that a few topology changes in information dissemination layer network will directly affect the critical value of infectious disease.

With the development of the network study, the scholars found out that there is an adaptive feedback process on the awareness information diffusion and the epidemic spreading. That is to say, the individual will break or generate their link with other individuals as the diffusion process happing on the network, and then the network topology will change which will react to the spreading process. Based on the above feedback mechanism, scholars gave out the adaptive epidemic spreading and the adaptive information diffusion. Hu et al [15] introduced the adaptive process into the information diffusion to describe the phenomenon the individual generate the link with another awareness node, and the results show the process of adaptive network evolution accelerates the spread of information on the internet. In the process of the epidemic spreading, susceptible individuals will reduce the number of the infectious link in order to avoid infecting the disease, and generate another link with susceptible individuals. Therefore, the research that regard the adaptive network as the basic network of infectious diseases has been paid much attention, and the study has found a lot of interesting and different results from the static network. In view of the network link, Gross et al [4] and Shaw et al. [5] introduced the SIS and SIRS epidemic model on the adaptive network, they assumed that the link between the susceptible node and infected node will break with probability $\omega$, and the susceptible node which breaks the link will rewire a new link with another susceptible node. The experimental results showed that there are three kinds of epidemic spreading states on adaptive network: disease free, shock and infection. These new 
phenomena are also verified in different models. For example, the crisis awareness introduced into the adaptive behavior could cause a large range of shocks in the infected rate and the network topology [6]. The related studies also found that the adaptive behavioral response will make the effect of the target immune more obvious [7], and the community structure causing in the evolution of the adaptive process makes the disease controlling time more important [8,9]. The above researches focus on the dynamic characteristics in the steady state, however, the exploration of the transient characteristics is very limited. Recently, Marceau et al [10] proposed a precise analysis method, and verified the accuracy of the study through the analysis of some transient characteristics (such as susceptible nodes and edges of the proportion of infected nodes). They found that the initial network topology determines the size of the bi-stable region. The results obtained by Fenichel et al [11] also showed that the adaptive behavior of human beings can significantly change the communication process, and understanding the person's contact and balance mechanism can effectively improve the cost-effectiveness of disease control and help health authorities to avoid unnecessary consequences. In view of the nodes, RisauGusman and Zanette et al. [12] considered the link breaking and link rewiring on the network. All of the above results showed the influence induced by the link breaking mechanism on the network topology and the state of the node. The study of the reconnection mechanism from the point of network nodes is mainly concerned with the study of the heterogeneity of link rewiring. Song et al [13] presented rewiring mechanism based on spatial distance by giving a distance measure to divide the nodes, and assumed the short distance rewiring probability is $1-\mathrm{p}$, and the long distance rewiring probability is $\mathrm{p}$. The experimental results show that disconnecting the long distance link and establishing short distance link can effectively suppress the infectious disease. Rattana et al [14] established a link rewiring radius according to the geographical network establishment, and gave out the link rewiring mechanism based on the spatial distance. Their results showed that the increase of the radius of the reconnection will slow down the spread of infectious disease. Tang et al. [15] assigned the rewiring probability based on the core edge structure of the network nodes, and the experimental results showed that the epidemic threshold is increased.

When the epidemic spreads on the network, the individual will get awareness about the disease through the information diffusion in the awareness network. During the process of the awareness diffusion, in order to get more infectious diseases, the unconscious individuals will establish the connection with the conscious nodes initiatively, and then disconnect the connection with the unconscious nodes. Conscious susceptible individuals will decrease the contact with infected nodes to reduce their infected probability, which means that the awareness susceptible individual will disconnect the infected node with a certain probability, and establish a connection with other susceptible individuals. In this process, except for the link breaking process between the infected node and the susceptible node, susceptible individuals will also reduce contact with susceptible nodes who are closely related to the infected individual, that is, the awareness individual will regard the susceptible node who is closely related to the infected node as a disconnected object with a certain probability. In addition, in the link breaking process, the probability of the different susceptible individuals to avoid the infected chance is different.

Based on the above phenomena, an epidemic spreading model on the adaptive dual networks is proposed. The model describes the impact of the adaptive awareness diffusion on the behavior changes when the epidemic spreads in the network. At the same time, our model introduces the new link breaking phenomenon into the adaptive epidemic spreading process, and considers the heterogeneous link breaking probability.

Compared with the previous research, we will make three improvements to the traditional adaptive network evolution process. Firstly, we generate two adaptive networks to study the interplay between the epidemic spreading and the awareness diffusion. Secondly, we study the influence of the new link breaking process according to the phenomenon that the susceptible node which is related to the infected node will be regard as the link breaking node when the epidemic spreads. Finally, as the probability belong to different susceptible individuals is different, we assign different edges breaking probability for each susceptible individuals, and study the Impact of heterogeneous link breaking on the epidemic spreading. Based on the three aspects above, this paper presents an epidemic spreading model on adaptive dual network. Our findings show that this adaptive mechanism in the diffusion dynamics remarkably affects the two interacting spreading dynamics. In addition, given the new link breaking in the epidemic spreading process and the heterogeneous link breaking probability, we study the effects of the two characteristics of the model, and find that these two factors can hinder the epidemic spreading.

The remained of this paper is organized as follows. In Section 2, the model we proposed is introduced in this section. We detail our experiments and present an analysis of the results in Section 3. Finally, the conclusion of this paper is given.

\section{The epidemic spreading model on the adaptive dual network}

To present our main results, we first describe the model of the adaptive dual network in general, then introduce the evolution regulars of the two networks, the heterogeneous link breaking probability and the new 
link breaking phenomenon.

The proposed dual adaptive networks consist of an adaptive awareness network and an adaptive contagion network. The evolution process on the adaptive dual networks is as shown in the Fig.1:

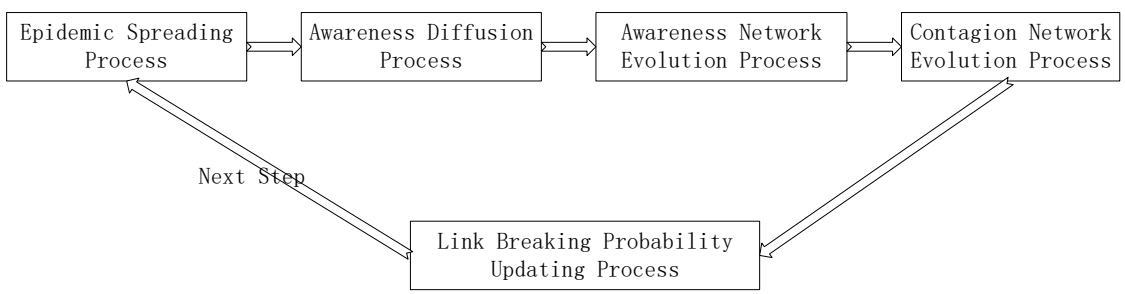

Figure.1 The evolution process on the adaptive dual network

On the physical contact network, each node has a certain initial disease state, namely the infectious state and the susceptible state. The initial state of each node in the awareness network is unconscious. The state of each node on the network will be affected by external factors. In the process of propagation, the asynchronous updating mode is adopted. Each step is divided into five parts:

\section{(1) Epidemic spreading process}

The states of the nodes in the contagion network are infected node (I), the susceptible node connected with infected node (SI) and the susceptible node disconnected with infected node (SS).

At each moment, the infected node $i$ will infect all neighbor nodes, and the neighbor node $\mathbf{j}$ will be infected with probability $\alpha_{j}$. If the neighbor node are stay in the S state, the node state will change to infected state I, and the node state will be updated. The conversion formula is as follows,

$$
\mathrm{S}_{\mathrm{i}}+\mathrm{I}_{\mathrm{j}} \stackrel{\alpha_{\mathrm{j}}}{\rightarrow} \mathrm{I}_{\mathrm{i}}+\mathrm{I}_{\mathrm{j}}
$$

Where, $\alpha_{j}$ represents the infected probability. The larger the value is, the easier the node will be influenced by the neighbor.

The infected node $\mathbf{j}$ will recover with probability $\beta_{\tilde{j}}$, and the conversion formula is as follows,

\section{(2) Awareness diffusion process}

$$
\mathrm{I}_{\mathrm{j}} \stackrel{\beta_{\mathrm{j}}}{\longrightarrow} \mathrm{S}_{\mathrm{j}}
$$

At each moment, the epidemic spreading in the contagion network results in the node corresponding to the infected node in the awareness network being aware of the epidemic. The awareness node $i$ will sent awareness to all the neighbor nodes, the neighbor node $\mathbf{j}$ will accept the awareness and be aware of the disease with probability $\delta_{\mathrm{j}}$. If the neighbor node state is $\mathrm{U}$ state, the node state will change to the $\mathrm{A}$ state. The conversion formula is as follows,

$$
\mathrm{U}_{\mathrm{i}}+\mathrm{A}_{\mathrm{j}} \stackrel{\sigma_{\mathrm{j}}}{\rightarrow} \mathrm{A}_{\mathrm{i}}+\mathrm{A}_{\mathrm{j}}
$$

Where, $\delta_{\mathrm{j}}$ represents the awareness probability. The larger the value is, the easier the node will be influenced by the neighbor.

The awareness node $\mathbf{j}$ will forget the information with probability $\lambda_{\mathrm{j}}$, and the conversion formula is as follows,

\section{(3) Awareness Network evolution}

$$
\mathrm{A}_{\mathrm{j}} \stackrel{\mathrm{X}_{\mathrm{j}}}{\longrightarrow} \mathrm{U}_{\mathrm{j}}
$$

The diffusion of the awareness among the crowd leads a part of node to be aware of the disease. The unawareness nodes will communicate with the awareness node in order to get more information to protect themselves. During this process, the network topology will evolve with the adaptive awareness evolution regular.

\section{(4) Contagion Network evolution}

The spread of the disease results in the individual to communicate with each other and to take some measures to protect themselves. The individual will reduce the chance to contact with the infected node which induces the topology changing. During this process, the network topology will evolve with the adaptive contagion evolution regular.

\section{(5) Rewiring process}

After the completion of the evolution of infectious disease network, it is necessary to update the link breaking probability as the topology of the network has changed. 


\section{AWARENESS NETWORK EVOLUTION REGULAR}

When the epidemic spreads on the network, the individual will get awareness about the disease through the information diffusion in the awareness network. During the process of the awareness diffusion, in order to get more infectious diseases, the unconscious individuals will establish the connection with the conscious nodes initiatively, and then disconnect the connection with the unconscious nodes.

\subsubsection{Unawareness link breaking}

The node which is unaware of the disease in the network will disconnect the unawareness node with probabilityt.

\subsubsection{Awareness link generating}

The unawareness node which break the link will rewire the link to an awareness node with probability

$\varphi$.

\subsection{Contagion Network evolution regular}

In the contagion network, conscious susceptible individuals will decrease the contact with infected nodes to reduce their infected probability, which means that the awareness susceptible individual will disconnect the infected node with a certain probability, and establish a connection with other susceptible individuals. In this process, except for the link breaking process between the infected node and the susceptible node, susceptible individuals will also reduce contact with susceptible nodes who are closely related to the infected individual, that is, the awareness individual will regard the susceptible node who is closely related to the infected node as a disconnected object with a certain probability.

\subsubsection{Contagion Link breaking}

The node on the contagion network will be aware of the disease after the diffusion of the awareness. The awareness node which is connected to the infected node will break the infected link with probability $\omega_{1}$, and the awareness node which is not connected to the infected node will break its link with the susceptible node connected with infected node with probability $\omega_{2}$.

\subsubsection{Link generating}

The node which breaks the link will rewire to the susceptible node which is not connected to the infected node with probability?.

We will describe the new link breaking phenomenon in the figure 3.

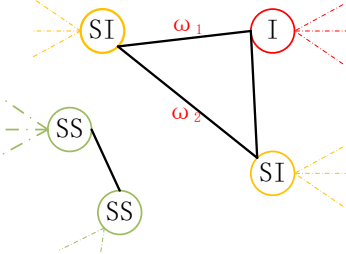

(a) Infected Link Breaking

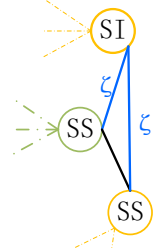

(b) Link Rewiring

SI

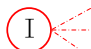

Figure.2 Contagion network evolution process

\subsection{Rewiring process regular}

After the evolution of the contagion network, the topology of the network changes, and the link breaking probability of the individual changes which leads to update the link breaking probability.

The $S_{I}-S_{S}$ link breaking probability belongs to the susceptible node $S_{S}$ which is not connected with the infected node is dependent on the intimate to the susceptible node $S_{I}$ which is connected to the infected node. In particular, we define the intimate as the infected intimate. The node which state is $\mathrm{S}_{\mathrm{I}}$ will become the link breaking object when the infected intimate is high. This description indicates that the $S_{I}-S_{S}$ link breaking probability is proportional to the infected intimate.

According to the above analysis, we will give out a parameter to measure the infected intimate, and then carry out a heterogeneous $S_{I}-S_{S}$ link breaking probability. The literature [29] has pointed out that the common friend between two nodes will increase the way of contact between the two nodes. For example, the common friend invited the two nodes to their birthday party. Therefore, we use the common friend number to describe the contact way which measures the intimate between the susceptible node connected to the infected node and the infected node.

In each step, the $\mathrm{S}_{\mathrm{I}}-\mathrm{S}_{\mathrm{S}}$ link breaking probability is calculating with the follow formula, 


$$
\omega_{2}=\mathrm{a} \cdot \frac{\mathrm{c}_{\mathrm{s}_{\mathrm{I}}}(\mathrm{t})+1}{\mathrm{k}_{\mathrm{I}}(\mathrm{t})}
$$

Where, $c_{s_{I} I}(\mathrm{t})$ represents the number of the contact way induced by the common friends between the susceptible node connected with infected node and the infected node. And $k_{I}(\mathrm{t})$ represents the degree of the infected node. In addition, 1 in the molecule represents the link between the infected node and the susceptible node.

According to the description of the two dynamic process, we will further explain the above process with the adaptive dual network epidemic figure. As shown in Fig. 3, the diffusion of the awareness will happen in the top network, and the spread of the disease will happen in the bottom network. During the awareness spreading process, the unawareness node 10 will break the link with node 4 with probability $\tau$ and generate a new link to the node 13 with probability $\varphi$. The diffusion of the awareness will influence the spreading of the disease which leads to the node in the contagion network be aware of the disease. In this figure, the node 7 will break the link to the node 5 with probability $\omega_{1}$, and generate a new link to the node 4 with probability $\zeta$. The node 6 which is aware of the node and not connected with the infected node will break the link to the node 7 with probability $\omega_{2}$, and generate a link to the node 11 .

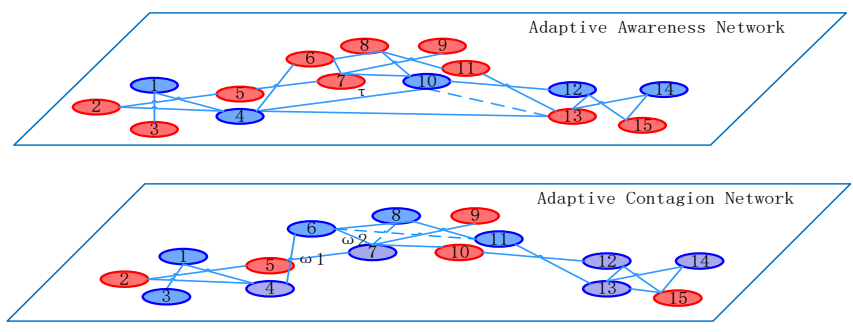

Figure. 3 Illustration of interplay spreading process on the adaptive dual network

\section{SIMULATION}

To better understand the effects of the adaptive awareness diffusion on the epidemic spreading, we investigate the effects of two key factors of the model: infected rate and epidemic threshold. To this end, we create dual adaptive networks for different experiments where individuals are represented by the vertices and their contacts are represented by links. The dual networks used in the experiments are given as following:

The contagion network is a scale free network consisted of 1000 node constructed by the BA network generation algorithm [28]. In our experiment, the exponent of the contagion network is 2.5 . Then, we add 400 links to the network randomly to generate the awareness network. Finally, we will select two nodes as the infected nodes. The evolution of the state of the network node is carried out until the density of infected nodes tends to be stable.

\subsection{The effect of the infected probability}

The infected probability is an important impact in the epidemic spreading process. In this section, we will discuss the transient evolution of infectious diseases under different infection rates.

To explore the effect of the infected probability, we plot this dynamic process on four different infected probability $\alpha: \alpha=0.2, \alpha=0.4, \alpha=0.6, \alpha=0.8$. The values of the other parameters are: $\beta=0.2, \lambda=0.5, \delta=0.2, \varepsilon=0.5, \omega_{1}=0.2, \theta=0.3$, respectively. The transient state difference between the homogeneous link breaking model and the heterogeneous link breaking probability is illustrated in Fig. 4. In addition, the red and blue curves are the infected rate changes with the time in the heterogeneous and homogeneous situations. By comparing the two panels, it is clear that the network heterogeneous link breaking probability will result in remarkable changes of the epidemic spreading. Fig. 4(a) suggests that the effect of infected probability on the infected rate can be neglected when the infected probability is smaller than the awareness probability and the infected probability is closely to the recovery probability. Fig. 4(b) shows the infected rate of the two model stay in steady state after transient growth when the infection probability is greater than the probability of recovery. Fig. 4(c) and Fig. 4(d) indicate that the value of infected probability has remarkable influence on the infected rate difference between the heterogeneous and the homogeneous model .That is to say, the difference disappears with the increase of the infected probability when the infection probability is greater than the awareness probability. This phenomenon can be explained as follows: when the infected probability is larger than the awareness probability, the diffusion of the disease is faster than the spreading of the awareness, which leads to less individual be aware of the disease and less individual will protect themselves by breaking the infected link. 

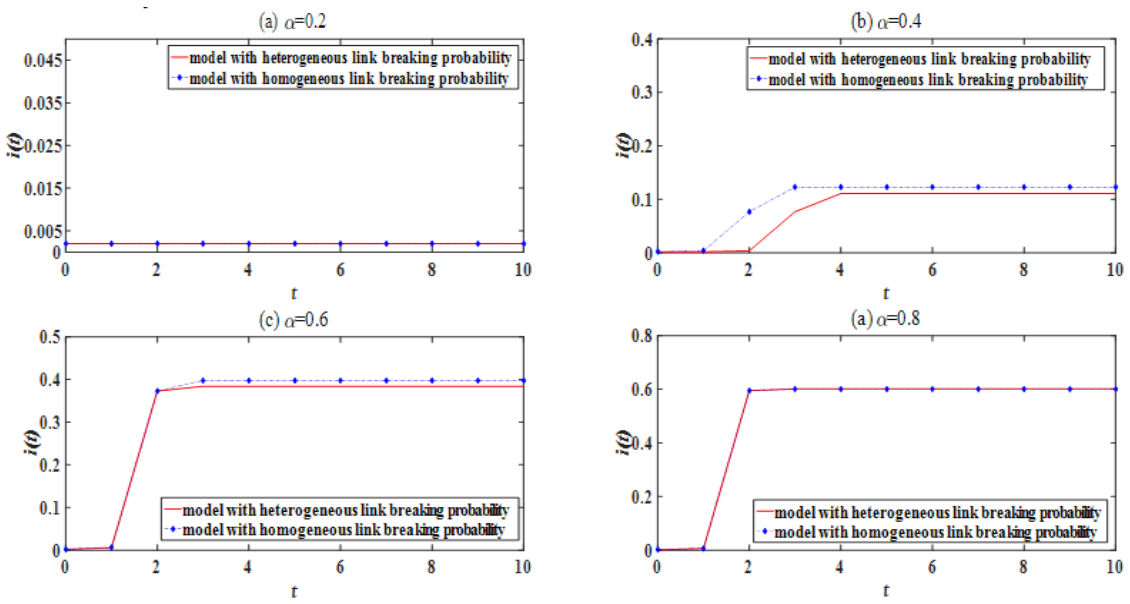

Figure. 4 The effect of the infected probability

The above analysis indicates that, in the early outbreak of infectious disease, the spread of awareness will lead individual to reduce contacting with the infected individuals which slows down the epidemic spreading and reduce the infected size. At the same time, the heterogeneity of the link breaking probability will suppress the spread of infectious diseases and reduce the scale of infectious diseases.

\subsection{The effect of the awareness probability}

In the proposed model, we propose an epidemic spreading model on the dual network, which is able to consider the process of awareness information dissemination in the evolution process of the single layer adaptive network. In this part, we further study the impact on the adaptive transmission of infectious diseases by changing the probability of awareness information dissemination.

In the Fig.5, we plot this dynamic process on four different awareness probability $\delta$ : $\delta=0.2, \delta=0.4, \delta=0.6, \delta=0.8$. The values of the other parameters are: $\beta=0.2, \lambda=0.5, \alpha=0.2, \varepsilon=0.5, \omega_{1}=0.2, \theta=0.3$, respectively. This figure shows that the transient process differences between the heterogeneous and homogeneous situations with different awareness probability, in which the red and green curves represent the infected rate evolution with the changing of the awareness probability in the heterogeneous and homogeneous link breaking model. Fig.5 indicates that the value of the awareness probability has remarkable influence on the infected rate, and the heterogeneous link breaking will induce the changing of the infected rate. As shown in the Fig. 5(d), when the infected probability is less than the awareness probability and the awareness probability is larger than the unawareness probability, the infected rate will increase rapidly and achieve a steady state. When the awareness probability is closely to the unawareness probability, the infected rates in the homogeneous and heterogeneous situations achieve a steady state after a slowly growth. The difference between the two dynamics is that the heterogeneous link breaking will slow down the epidemic spreading and reduce the infected size, and the difference between the two dynamics is increasing with the time changing.
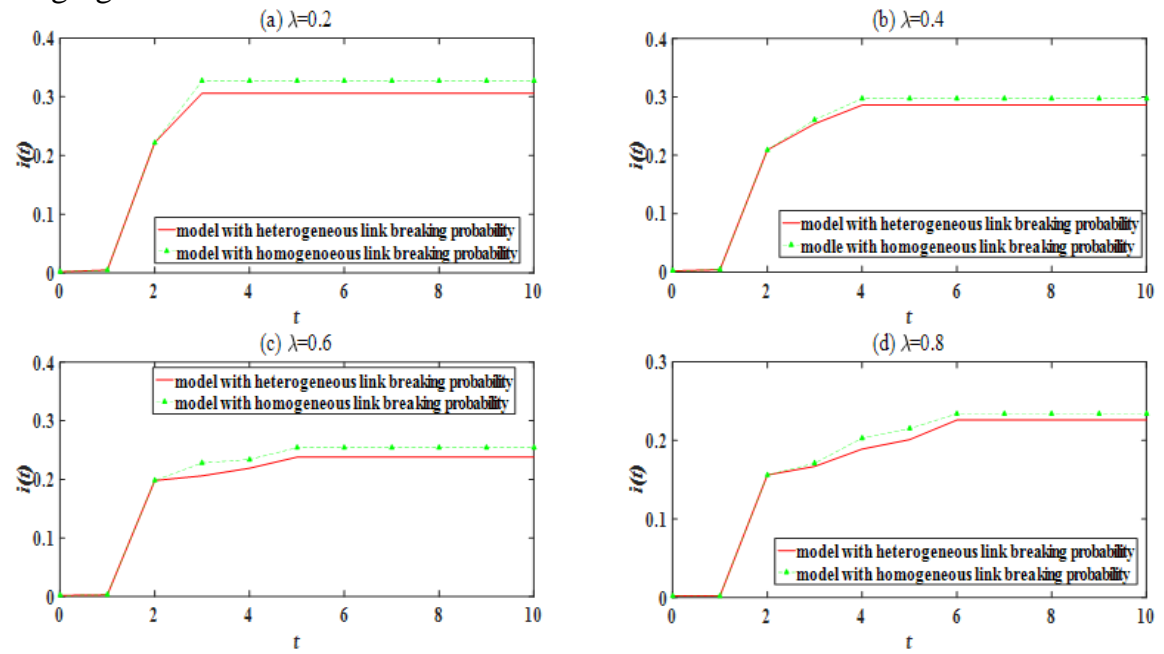

Figure. 5 The effect of the awareness probability

The results of data analysis show that with the increase of the awareness probability, the infected sizes in the two model are reducing and the difference between the two situations is reducing. Therefore, in the 
prevention of infectious diseases, the ways by increasing the media to increase the awareness of individual can reduce the scale of infectious diseases.

\subsection{The awareness probability of the infected node effect}

The source of the awareness is the awareness of the infected individual. Therefore, we implement the impact of the infected node awareness probability. The experiment results are shown in the Fig. 6 in which we plot this dynamic process on three different awareness probability $\lambda: \lambda=0.2, \lambda=0.4, \lambda=0.5$. The values of the other parameters are: $\beta=0.2, \delta=0.2, \alpha=0.4,=0.2, \theta=0.3$, respectively. In this figure, the Fig. 6 (a) and the Fig. 6 (b) represent the infected rate in the heterogeneous and homogeneous situation, respectively. From the Fig. 6 (a), the different awareness probability belongs to the infected node will lead to different process. When the awareness probability is smaller than the infected probability, the infected rate will not change. This is because of the speed of the epidemic is faster than that of the awareness, the increasing of the awareness probability belong to the infected node has no influence on the epidemic spreading. When the awareness probability becomes large, the infected rate will decrease with the awareness probability belong to the infected node increases. In particular, the faster the awareness probability increases, the larger the range of the infected rate reduce. Compared Fig. 6(a) with Fig. 6(b), we can also see that the heterogeneous link breaking will reduce the epidemic size, and the influence induced by the heterogeneous will decrease when the infected node awareness becomes small.
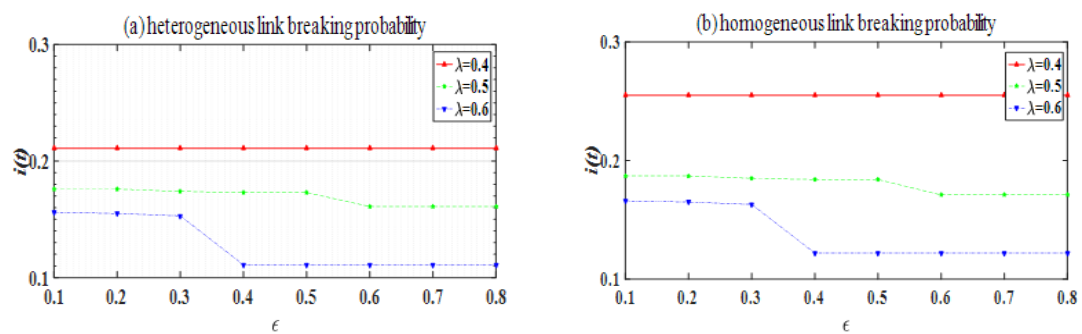

Figure. 6 The effect of the infected node awareness probability

According to the above analysis, we can observe that when infected consciousness spread faster than the spread of infectious diseases, the infected node awareness probability will increase, and then reduce the epidemic size. In addition, the heterogeneous disease link disconnection probability will weaken the epidemic scale, its influence will increase along with the weakening of the infected node awareness probability.

\subsection{The effect of the $S_{I}-S_{S}$ link breaking}

In this model we improve the traditional model by considering a new link breaking phenomenon. Therefore, it is necessary to compare the model with the model without considering the $\mathrm{S}_{\mathrm{I}}-\mathrm{S}_{\mathrm{S}}$ link breaking.

According to the different infected probability, we draw the $(t-i(t))$ curves in different infected probability. As shown in the Fig. 7, we assume that the value of the parameters are (a) $\alpha=0.2$, (b) $\alpha=0.4$, (c) $\alpha=0.6$, (d) $\alpha=0.8$. The values of the other parameters are: $\beta=0.2, \lambda=0.5, \alpha=0.2, \varepsilon=0.5, \omega_{1}=0.5, \theta=0.3$, respectively.
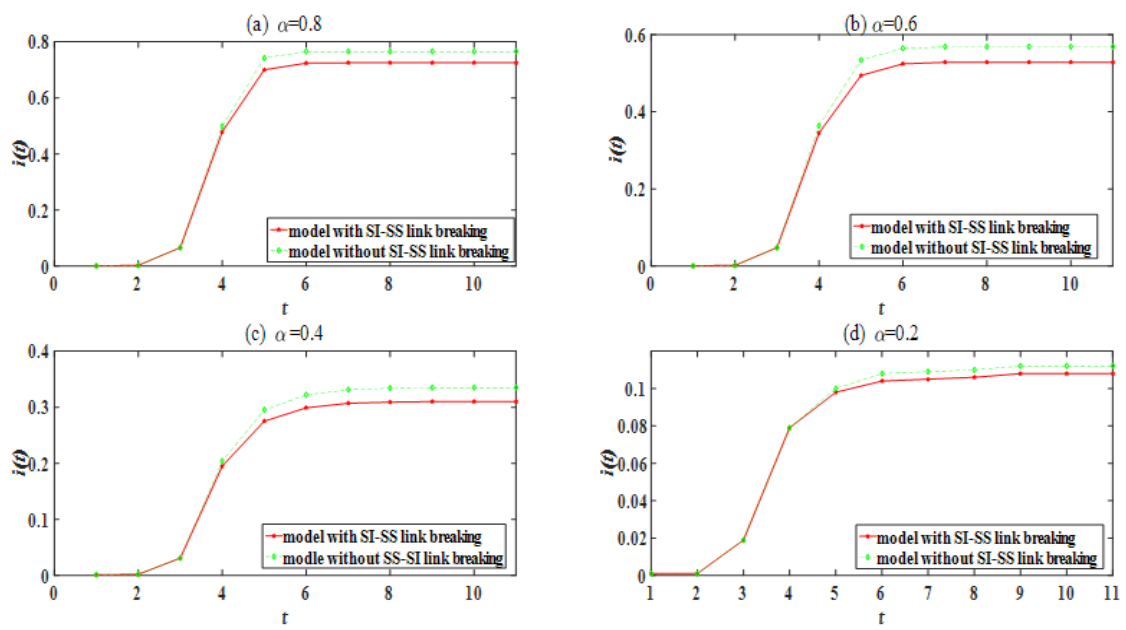

Figure. 7 The effect of $\mathrm{S}_{\mathrm{I}}-\mathrm{S}_{\mathrm{S}}$ link breaking

In the Fig. 7, the green dotted line represents the infected rate curves with the evolution time without considering the $\mathrm{S}_{\mathrm{I}}-\mathrm{S}_{\mathrm{S}}$ link breaking, the red line indicates the infected rate curves with the evolution time 
considering the $\mathrm{S}_{\mathrm{I}}-\mathrm{S}_{\mathrm{S}}$ link breaking.

From the two panels, we can observe that the infected rate rapidly increases with the time evolution and achieve a steady state. For example, in the Fig. 7(a) the red line and the green dashed line change with time. For the speed of infectious diseases spreading, the speed of epidemic spreading in the model considering the $\mathrm{S}_{\mathrm{I}}-\mathrm{S}_{\mathrm{S}}$ link breaking will slow than that in the model without considering the link breaking. For example, in Fig. 7 (b), the slope of the red line is less than the slope of the green dashed line.

\subsection{The effect of the dual adaptive network}

The model we proposed considers the awareness diffusion and the epidemic spreading by constructing an adaptive dual network. In this section, we will discuss the influence of the dual adaptive networks.

Fig. 8 shows the epidemic spreading process with different infected probability. The red curve represents the infected rate in the dual adaptive network, and the green curve represents the infected rate in the static dual network. Now, we assume that (a) $\alpha=0.2$,(b) $\alpha=0.4$,(c) $\alpha=0.6$,(d) $\alpha=0.8$. The values of the other parameters are: $\beta=0.2, \lambda=0.5, \alpha=0.2, \varepsilon=0.5, \omega_{1}=0.2, \theta=0.3$, respectively.
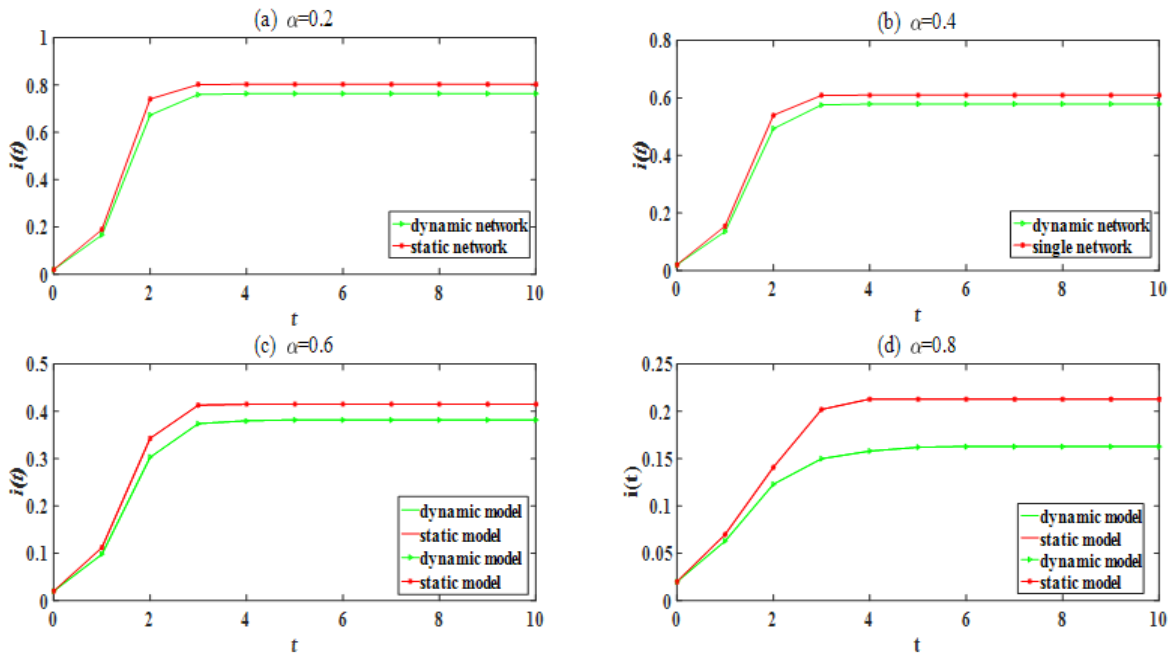

Figure. 8 The effect of the adaptive dual networks

In the Fig. 8, when the infected probability is different, the epidemic spreading process is same. The infected rate will increases with the time evolves. The infected rate increases fast when the infected time is short. But the increasing range in the adaptive network is smaller than that in the static model. As shown in Fig. 8(c), the green slope was significantly greater than that of the red curve. When the spread time increases, the infected rate achieves a steady state. And the infected rate of the adaptive network is smaller than that of the static model. As shown in Fig. 8(d), the infected rate belongs to the green line is 0.17 , and the infected rate in the red line is 0.22 .

From the above analysis, we can see that the dynamic dual network slows down the spread of infectious diseases and reduces the spread of infectious diseases. The reason is that the adaptive network increases the awareness of the individual and reduces the chance connected to the infected node.

\section{CONCLUSION}

As summary, in this paper, we have carried out an epidemic spreading model on the adaptive dual networks through considering the adaptive mechanism in the diffusion of disease and awareness. With the help of the adaptive dual networks, we are able to model the interplay between these two kinds of adaptive dynamics processes. Afterwards, we give a mechanism to measure the heterogeneous link breaking probability. In addition, we consider a new link breaking phenomenon in the adaptive epidemic spreading process which means the susceptible node connected to the infected node will be a link breaking node. From the above three parts, we study the interplay between two adaptive dynamics. According to the transient and steady state analysis, we display the influences of the infected probability, the awareness probability, the infected node awareness probability, the $\mathrm{S}_{\mathrm{I}}-\mathrm{S}_{\mathrm{S}}$ link breaking and the adaptive network on the epidemic spreading. Compared with the static dual networks with the same dynamics, we find that the adaptive process increase the spreading of the awareness which directly affect the epidemic threshold. These results show the importance of taking the adaptive information spreading process into account when we try to control the spread of epidemics. From the point of view of heterogeneous link breaking probability, our results show that the heterogeneous link breaking probability will suppress the epidemic spreading and reduce the epidemic size. 


\section{ACKNOWLEDGEMENTS}

We thank the anonymous referees for helpful comments and constructive suggestions. This work was partially sponsored by the National Nature Science Foundation of Chain (Project No. 61401015,71540015), the Foundation of the BUCEA (Project No. ZF15069)

\section{REFERENCE}

[1]. Z.K. Istvan, C Jackie, R Mario, L.S. Péter, The impact of information transmission on epidemic outbreaks, Mathematical Biosciences.225, 2010, 1-10.

[2]. D.S Faryad, A.C Fahmida, M.S Caterina. On the existence of a threshold for preventive behavioral responses to suppress epidemic spreading, Science Report, 2, 2012, 632.

[3]. Q.C Wu, X.C Fu, M Small, X.J Xu, The impact of awareness on epidemic spreading in networks, Chaos, 22, 2012, 013101

[4]. Z.Y Ruan. M Tang, Z.H Liu, Epidemic spreading with information-driven vaccination, Physical Review E Statistical Nonlinear \& Soft Matter Physics, 86, 2012, 036117.

[5]. H.J Hang, T.M Hie, K.B. Seung, Immunization dynamics on a two-layer network model, Physical A, 361, 2006, 534-542.

[6]. F Sebastian, G Erez, A.A.J Vincent, Epidemic disease, awareness, and local behavioral response, Journal of Theoretical Biology, 264, 2010, 501-509.

[7]. C Granell, S Gomez, A Arenas, Dynamical interplay between awareness and epidemic spreading in multiplex networks, Physical Review Letter, 111, 2013,128701.

[8]. Q.T Guo, X Jiang, Y.J Lei, Z.M Zheng, Two-stage effects of awareness cascade on epidemic spreading in multiplex network, Physical Review E, 91, 2015,012822.

[9]. T Liu, P Li et.al, Community size effects on epidemic spreading in multiplex social networks, Plos One, 11, 2016, e0152021.

[10]. C Granell, S Gomez, A Arenas, Competing spreading processes on multiplex networks, Physical Review E, 90, 012808(2013).

[11]. W Wang, M Tang, Impacts of complex behavioral responses on asymmetric interacting spreading dynamics in multiplex networks, Science Report, 6, 2015, 25617.

[12]. Q.T Guo, X Jiang et.al, The Role of node heterogeneity in the coupled spreading of epidemics and awareness, Plos One, 11, 2016, e0161037

[13]. W Wang, M Tang, H Yang, Asymmetrically interacting spreading dynamics on complex layered networks, Scientific Reports, 4, 2014.

[14]. Q.T Guo, X Jiang, Y.J Lei, Z.M Zheng, Epidemic spreading with activity-driven awareness diffusion on multiplex network, Chaos, 26, 2016, 043110.

[15]. K Hu, Y Tang, Information diffusion on adaptive network, Chinese Physics B, 2008, 17(10):3536-3541

[16]. T Gross, J.D Carlos, B Blasius, Epidemic dynamics on an adaptive network, Physical Review Letters, 20, 2006, 208701

[17]. L.B Shaw, I.B Schwartz, Fluctuating epidemics on adaptive networks, Physical Review Letters, 77, 2008, 066101

[18]. T Gross, G.K Ioannis, Robust oscillations in SIS epidemics on adaptive networks: Coarse-graining by automated moment closure, Europhysics Letters, 82, 2008, 38004.

[19]. L.B Shaw, I.B Schwartz, Enhanced vaccine control of epidemics in adaptive networks, Physical Review E, 81, 2010, 046120.

[20]. H Yang, M Tang, H.F Zhang, Efficient community-based control strategies in adaptive networks, Physics, 14, 2012, 123017.

[21]. H.K Liu, H Yang, M Tang, T Zhou, Local transient-based quarantine strategy in adaptive networks, Science Sinica, 2014.

[22]. M Vincent, P.A Noël, H.D Laurent, et al. Adaptive networks: Coevolution of disease and topology, Physical Review E, 3, 2010,036116.

[23]. F Elil, C.C Carlos, G.C Michele, Cristina Villalobos. Adaptive human behavior in epidemiological models, Process National Academia Science USA, 15, 2011, 6306-6311.

[24]. S Risau, D Zanette, Contact switching as a control strategy for epidemic outbreaks, Journal of Theoretical Biology, 52, 2009.

[25]. Y.R Song, G.P Jiang, Y.W Gong, Epidemic propagation on adaptive co-evolutionary networks with preferential local-world reconnecting strategy, Chinese Physics B, 22, 2013, 040205.

[26]. R Prapanporn, L Berthouze, Z.K Istvan, The impact of constrained rewiring on network structure and node dynamics, Physical Review E, 90, 2014, 052806 
[27]. H Yang, M Tang, S.M Cai, T Zhou, Core-periphery structure in heterogeneous network adaptive network and its inhibiting effect on epidemic spreading, Chinese Physical Society, 65, 2016, 058901.

[28]. L.B Albert, R Albert. Emergence of scaling in random networks, Science, 286, 1999, 509-512.

[29]. H Huang, Z.J Yan, Y.H Pan, Measuring importance to improve immunization performance. Physical A, 9, 2014. 